\title{
2-Adic Arithmetic-Geometric Mean and Elliptic Curves
}

\author{
Kensaku KINJO* and Yuken MIYASAKA* \\ Mathematical Institute, Tohoku University, Sendai 980-8578, Japan
}

Received January 29, 2009; final version accepted May 21, 2009

\begin{abstract}
We prove that the ratio of the sequences of the arithmetic geometric mean over a finite extension of $\mathbb{Q}_{2}$ is periodically convergent, and that the limit is related to the canonical lift of an elliptic curve defined over a finite field of characteristic two.
\end{abstract}

KEYWORDS: arithmetic-geometric mean, canonical lift, elliptic curve

\section{Introduction}

We begin with a review of the arithmetic-geometric mean defined over $\mathbb{R}$. Let $a$ and $b$ be positive real numbers. We define two sequences $\left\{a_{n}\right\}_{n \geq 0}$ and $\left\{b_{n}\right\}_{n \geq 0}$ inductively by

$$
\begin{array}{ll}
a_{0}:=a, & b_{0}:=b, \\
a_{n+1}:=\frac{a_{n}+b_{n}}{2}, & b_{n+1}:=\sqrt{a_{n} b_{n}}(n \geq 0),
\end{array}
$$

where $b_{n+1}$ is the positive square root of $a_{n} b_{n}$. These are called the sequences of the arithmetic-geometric mean of $a$ and $b$, and converge to a common limit (see, Cox [4]). Gauss discovered amazing depth of this subject, including a connection between the arithmetic-geometric mean and elliptic integrals. (We will touch a few aspects of his works in Appendix A.)

Henniart and Mestre in [7] defined the arithmetic-geometric mean over a $p$-adic field for all primes $p$, which is called the $p$-adic arithmetic-geometric mean, and related it with the period of an elliptic curve having multiplicative reduction over the $p$-adic field. Unfortunately, unless $p=2$, their method cannot be generalized to elliptic curves with good reduction, essentially because the isogeny which connects the arithmetic-geometric mean with elliptic curves, appearing in Section 2, is of degree 2.

Still, it is possible to use the 2-adic arithmetic-geometric mean in a study of elliptic curves with good ordinary reduction over a 2-adic field. It is explained in [6] and [9] that, when an ordinary elliptic curve $\tilde{E}$ over a finite field of characteristic two is given, one can exploit the 2-adic arithmetic-geometric mean in order to compute the canonical lift of $\tilde{E}$.

They, however, worked over an unramified extension of $\mathbb{Q}_{2}$, because the canonical lift is defined over the unramified field. We present in this paper that their method can be modified in such a way that it works over any (possibly ramified) finite extension of $\mathbb{Q}_{2}$.

In order to state our results, we introduce the 2-adic arithmetic-geometric mean. Let $K$ be a finite extension of $\mathbb{Q}_{2}$ and $v$ the valuation of $K$ satisfying $v(2)=1$. Note that, for a non-zero element $\xi \in K$ satisfying $v(\xi-1)>2$, there exists a unique element $\eta \in K$ such that $\eta^{2}=\xi$ and $v(\eta-1)>1$. We write $\sqrt{\xi}$ for $\eta$. Let $a$ and $b$ be non-zero elements of $K$ satisfying $v((a / b)-1) \geq 3$. We define the sequences $\left\{a_{n}\right\}_{n \geq 0}$ and $\left\{b_{n}\right\}_{n \geq 0}$, which is called the sequences of the 2-adic arithmetic-geometric mean of $a$ and $b$, in a way similar to (1.1):

$$
\begin{array}{ll}
a_{0}:=a, & b_{0}:=b, \\
a_{n+1}:=\frac{a_{n}+b_{n}}{2}, & b_{n+1}:=b \sqrt{\frac{a_{n}}{b_{n}}}(n \geq 0) .
\end{array}
$$

Note that $v\left(\left(a_{n} / b_{n}\right)-1\right) \geq 3$ implies $v\left(\left(a_{n+1} / b_{n+1}\right)-1\right) \geq 3$ and that $v\left(\left(a_{n} / b_{n}\right)-1\right)=3$ implies $v\left(\left(a_{n+1} / b_{n+1}\right)-\right.$ $1)=3$, hence the definition of $b_{n+1}$ for each $n \geq 0$ makes sense. Unlike the case of $\mathbb{R}$ recalled in the beginning, the sequences $\left\{a_{n}\right\}_{n>0}$ and $\left\{b_{n}\right\}_{n>0}$ do not always converge. In fact, these sequences converge if and only if $v((a / b)-1)>$ 3 (for the proof, see [7]). When $v((a / b)-1)>3$, these sequences converge to a common limit. On the other hand, when $v((a / b)-1)=3$, we can define the sequences $\left\{a_{n}\right\}_{n \geq 0}$ and $\left\{b_{n}\right\}_{n \geq 0}$ although they do not converge. We consider mainly the case $v((a / b)-1)=3$. Let $\mu_{n}:=a_{n} / b_{n}$ be the ratio of these sequences, which has the relation

* Corresponding author. E-mail: sa6m16@math.tohoku.ac.jp; sa7m27@math.tohoku.ac.jp 
$\mu_{n+1}=\left(\mu_{n}+1\right) / 2 \sqrt{\mu_{n}}$. The object of this paper is to give a proof of the following theorem, which gives the extension of the results ([6], [9]) in the case that $K$ is unramified over $\mathbb{Q}_{2}$.

Theorem 1.1. Let $K$ be a finite extension of $\mathbb{Q}_{2}$ and $d$ the residue degree of $K$. For $a, b \in K$ with $v((a / b)-1)=3$, let $\left\{a_{n}\right\}_{n \geq 0}$ and $\left\{b_{n}\right\}_{n \geq 0}$ be the sequences of the 2-adic arithmetic-geometric mean of $a$ and $b$, and put $\mu_{n}:=a_{n} / b_{n}$ for each $n \geq 0$. Let $i \in\{0,1, \ldots d-1\}$.

1. The sequence $\left\{\mu_{d n+i}\right\}_{n \geq 0}$ is convergent in $K$. (We write $\mu_{i}^{\uparrow}$ for the limit of the sequence $\left\{\mu_{d n+i}\right\}_{n \geq 0}$.)

2. For each $n \geq 0$, the elliptic curve $E_{\mu_{n}^{2}}$ has good ordinary reduction, and $E_{\left(\mu_{i}^{\uparrow}\right)^{2}}$ gives the canonical lift of the reduction of $E_{\mu_{i}^{2}}$, where $E_{\lambda}$ is an elliptic curve defined by the equation

$$
E_{\lambda}: y^{2}=x(x-1)(x-\lambda) \text {. }
$$

This paper is organized as follows: In Section 2, we study an isogeny between elliptic curves having good ordinary reduction over a 2-adic field. Section 3 is devoted to the preparation for proving Theorem 1.1, thus the reader may omit this section. In Section 4 we prove the main theorem, and in Section 5 we conclude with a relation between elliptic curves defined over a 2-adic field and the 2-adic arithmetic-geometric mean. In Appendix A, we present a way of calculating the periods of an elliptic curve defined over $\mathbb{R}$ by using the arithmetic-geometric mean, and Appendix B describes a relation between the Hodge-Tate periods of a Tate curve and the $p$-adic arithmetic-geometric mean, which is a $p$-adic analogue of the theory presented in Appendix A.

Throughout $\S 2-\S 5, K$ is a finite extension of $\mathbb{Q}_{2}$, and $v$ is the valuation of $K$ satisfying $v(2)=1$. Let $\mathbb{F}$ be the residue field of $K$ and $d$ the extension degree of $\mathbb{F}$ over $\mathbb{F}_{2}$. We fix algebraic closures $\bar{K}$ and $\overline{\mathbb{F}}$ of $K$ and $\mathbb{F}$.

\section{Arithmetic-Geometric Mean and Isogeny}

We present a key lemma which connects the 2-adic arithmetic-geometric mean with the canonical lift. Let $\left\{a_{n}\right\}_{n \geq 0}$ and $\left\{b_{n}\right\}_{n \geq 0}$ be the sequences of the 2-adic arithmetic-geometric mean of $a$ and $b$, where $a, b \in K$ satisfy $v((a / b)-1)=3$. If we put $\mu_{n}:=a_{n} / b_{n}$ and $E_{n}:=E_{\mu_{n}^{2}}$, then there exists an isogeny

$$
g_{n}: E_{n} \rightarrow E_{n+1}, \quad(x, y) \mapsto\left(\frac{\left(x+\mu_{n}\right)^{2}}{4 \mu_{n} x}, \frac{y\left(x^{2}-\mu_{n}^{2}\right)}{8\left(\sqrt{\mu_{n}}\right)^{3} x^{2}}\right)
$$

of degree two.

Lemma 2.1. Let $E_{n}$ and $g_{n}$ be as above for each $n \geq 0$.

1. The elliptic curve $E_{n}$ has good ordinary reduction over $K$.

2. Let $\tilde{E}_{n} / \mathbb{F}$ be the reduced curve of a minimal Weierstrass model for $E_{n} / K(n \geq 0)$. Then $\tilde{E}_{n+1}$ is isomorphic to $\tilde{E}_{n}^{(2)}$ over $\mathbb{F}$, where $\tilde{E}_{n}^{(2)}$ is the 2-nd power Frobenius of $\tilde{E}_{n}$.

3. The following diagram commutes

$$
\begin{array}{ccc}
E_{n}(\bar{K}) & \stackrel{g_{n}}{\longrightarrow} & E_{n+1}(\bar{K}) \\
\text { red } \downarrow & & \text { red } \downarrow \\
\tilde{E}_{n}(\overline{\mathbb{F}}) & \stackrel{\mathrm{Fr}_{2}}{\longrightarrow} & \tilde{E}_{n}^{(2)}(\overline{\mathbb{F}}),
\end{array}
$$

where the vertical and lower maps are the reduction and 2-nd power Frobenius maps, respectively.

Proof. We put

$$
\gamma_{n}:=\frac{1+\mu_{n}^{2}+\sqrt{\left(\mu_{n}^{2}-1\right)^{2}+\mu_{n}^{2}}}{3} .
$$

The change of variables

$$
(x, y) \mapsto\left(\frac{x-\gamma_{n}}{4}, \frac{y-\left(x-\gamma_{n}\right)}{8}\right)
$$

transforms $E_{n}$ to

$$
E_{n}^{(m)}: Y^{2}+X Y=X^{3}+A_{n} X^{2}+B_{n},
$$

where

$$
A_{n}=\frac{3 \gamma_{n}-\left(1+\mu_{n}^{2}\right)-1}{2^{2}} \quad \text { and } \quad B_{n}=\frac{\left(\gamma_{n}-1\right)\left(\gamma_{n}-\mu_{n}^{2}\right) \gamma_{n}}{2^{6}} .
$$

Note that an elliptic curve

$$
y^{2}+x y=x^{3}+c, \quad \text { for some } c \in \mathbb{F}^{*}
$$


is ordinary, and that its $j$-invariant is $1 / c$. Therefore $E_{n}^{(m)}$ gives a minimal Weierstrass model of $E_{n}$ because

$$
v\left(A_{n}\right)>0 \text { and } v\left(B_{n}\right)=0,
$$

that is, the elliptic curve $E_{n}$ has good ordinary reduction. Moreover, we have

$$
B_{n+1} \equiv B_{n}^{2}(\bmod \pi),
$$

where $\pi$ is a prime element of $K$. This congruence implies that $\tilde{E}_{n+1}$ is isomorphic to $\tilde{E}_{n}^{(2)}$ over $\mathbb{F}$. We omit the proof of the commutativity of the diagram.

Remark. Using the above lemma, we explain the relation of the canonical lift to the 2-adic arithmetic-geometric mean. Let $\mu_{n}, E_{n}$, and $g_{n}: E_{n} \rightarrow E_{n+1}$ be as above for each $n$. By comparing (2.2), we obtain the following commutative diagram:

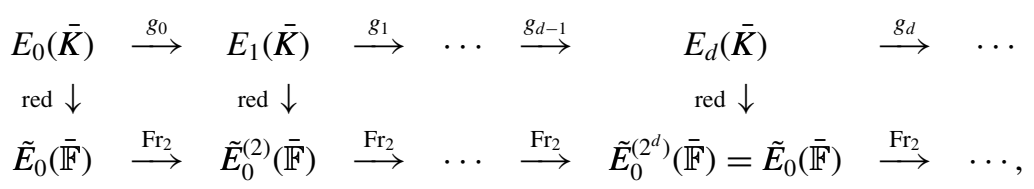

where $d:=\left[\mathbb{F}: \mathbb{F}_{2}\right]$. Note that, for each $n \geq 0$ and each $0 \leq i \leq d-1$, the reduction of $E_{d n+i}$ is isomorphic to $\tilde{E}_{0}^{\left(2^{i}\right)}$ over $\mathbb{F}$, and that the isogeny $g_{d(n+1)+i-1} \circ \cdots \circ g_{d n+i}$ is a lift of the Frobenius endomorphism on $\tilde{E}_{0}^{\left(2^{i}\right)}$. Therefore if the sequence $\left\{\mu_{d n+i}\right\}_{n \geq 0}$ converges to an element $\mu_{i}^{\uparrow}$, the elliptic curve $E_{\left(\mu_{i}^{\uparrow}\right)^{2}}$ should give the canonical lift of $\tilde{E}_{0}^{\left(2^{i}\right)}$ by the characterization of Serre-Tate [8] (stated below) because the endomorphism ring of $E_{\left(\mu_{i}^{\uparrow}\right)^{2}}$ contains the lift of the Frobenius endomorphism on $\tilde{E}_{0}^{\left(2^{i}\right)}$.

At the end of this section we state a characterization of the canonical lift by Serre-Tate. Let $R:=W(\mathbb{F})$ be the ring of Witt vectors over $\mathbb{F}$ and $F$ the fractional field of $R$, which is regarded as a subfield of $K$.

Theorem 2.2 (Serre-Tate). Let $\tilde{E}$ be an ordinary elliptic curve over $\mathbb{F}$. Then, there exists an elliptic curve $E^{\uparrow}$ defined over $F$, unique up to $F$-isomorphism, such that

1. The reduction of $E^{\uparrow} / F$ is isomorphic to $\tilde{E} / \mathbb{F}$ over $\mathbb{F}$,

2. $\operatorname{End}\left(E^{\uparrow}\right) \cong \operatorname{End}(\tilde{E})$.

This elliptic curve $E^{\uparrow} / F$ is called the canonical lift of $\tilde{E} / \mathbb{F}$.

\section{Elliptic Curves with Good Ordinary Reduction over a 2-adic Field}

The following proposition is proved by R. Carls ([1]) when $K$ is unramified. We prove it for any finite extension $K$ of $\mathbb{Q}_{2}$.

Proposition 3.1. Let $E$ be an elliptic curve having good ordinary reduction defined by

$$
E: y^{2}=x(x-\alpha)(x-\beta), \quad \alpha, \beta \in K,
$$

where the point $(0,0)$ lies in the kernel of the reduction map $\rho: E(\bar{K}) \rightarrow \tilde{E}(\overline{\mathbb{F}})$. Then $\alpha$ and $\beta$ satisfy $v((\alpha / \beta)-1)=4$. Moreover these elements are square in $K$.

Proof. We first remark that $v((\alpha / \beta)-1)=4$ implies $\alpha / \beta \in K^{* 2}$. Let $\lambda:=\alpha / \beta$. Note that $\lambda$ remains unchanged when the model of $E$ is replaced by a change of variables $(x, y) \mapsto\left(w^{2} x, w^{3} y\right)$ for some $w \in K^{*}$. Assume that the valuation of the discriminant of the given model of $E$ is zero, since $E$ has good ordinary reduction over $K$. This model, however, is not a minimal model of $E$ because the characteristic of the residue field of $K$ is two.

Thus we may suppose that a change of variables

$$
(x, y) \mapsto\left(u^{2} x+r, u^{3} y+u^{2} s x+t\right), \quad u, r, s, t \in K^{*} \text { and } v(u)=0
$$

gives an isomorphism to a minimal model of $E$. Then it follows that $v(r)<0$ because the point $(0,0) \operatorname{lies}$ in $\operatorname{Ker}(\rho)$. On the other hand, it follows that $v\left(u^{2} \alpha+r\right) \geq 0$ and $v\left(u^{2} \beta+r\right) \geq 0$ because the points $(\alpha, 0)$ and $(\beta, 0)$ do not lie in $\operatorname{Ker}(\rho)$. Thus we have $v(r)=v(\alpha)=v(\beta)$, and $v(\lambda)=0$. Now the $j$-invariant of $E$ is

$$
j(E)=2^{8} \frac{\left((\lambda-1)^{2}+\lambda\right)^{3}}{\lambda^{2}(\lambda-1)^{2}} .
$$

Since its valuation is zero, we conclude that $v(\lambda-1)=4$.

To prove $\alpha, \beta \in K^{* 2}$, we first show the following equivalences:

$$
\begin{aligned}
i \in K & \Longleftrightarrow E[4]^{c} \subset E(K) \\
& \Longleftrightarrow[2]_{E}^{-1}(0,0)(K) \neq \emptyset,
\end{aligned}
$$


where $i$ is a primitive 4 -th root of unity, $E[4]^{c}:=E[4] \cap \operatorname{Ker}(\rho)$, and $[2]_{E}^{-1}(0,0)(K)$ means the set $\{P \in E(K) ; 2 P=$ $(0,0)\}$. Note that we have an isomorphism as $\operatorname{Gal}\left(\bar{K} / K^{u r}\right)$-module

$$
E[4]^{c} \cong \mu_{4, \bar{K}},
$$

where $K^{u r}$ is the maximal unramified extension of $K$, because $E$ has good ordinary reduction over $K$.

The first equivalence (3.1) is given as follows: Assume $i \in K$. Then the isomorphism (3.3) means that $K\left(E[4]^{c}\right)$ is a finite unramified extension of $K$. However, if $\left[K\left(E[4]^{c}\right): K\right]>1$, this extension is ramified because $E[4]^{c}$ is contained in $\operatorname{Ker}(\rho)$. Therefore $\left[K\left(E[4]^{c}\right): K\right]=1$. Conversely we assume $E[4]^{c} \subset E(K)$. Then the isomorphism (3.3) means that there exists a finite unramified extension $L$ of $K$ such that $i \in L$. However, if $[K(i): K]>1$, this extension is ramified. Thus $[L: K]=1$.

The second equivalence (3.2) is given as follows: Assume $E[4]^{c} \subset E(K)$. Then a generator of $E[4]^{c}$ lies in $[2]_{E}^{-1}(0,0)(K)$. Conversely if we take a point $P \in[2]_{E}^{-1}(0,0)(K)$, the point $P$ or $P+Q$ is a generator of $E[4]^{c}$, where $Q=(\alpha, 0) \in E(K)$ is a point of order 2 which does not lie in $\operatorname{Ker}(\rho)$.

Finally we consider two cases.

Case 1. $i \in K$. For a point $P=(x, y) \in[2]_{E}^{-1}(0,0)(\bar{K})$, we have

$$
x= \pm \beta \sqrt{\frac{\alpha}{\beta}}, \quad y^{2}=-\alpha \beta^{2}\left(\sqrt{\frac{\alpha}{\beta}} \mp 1\right)^{2}
$$

because of the duplication formula of elliptic curves. By (3.1) and (3.2), we may assume $x, y \in K$. Since $\alpha / \beta \in K^{* 2}$, we obtain $\alpha, \beta \in K^{* 2}$.

Case 2. $i \notin K$. Put $L:=K(i)$. Applying (3.1) and (3.2) to $L$, we obtain $[2]_{E}^{-1}(0,0)(L) \neq \emptyset$. For $P=(x, y) \in$ $[2]_{E}^{-1}(0,0)(L)$, we also have (3.4). Hence we see $-\alpha \in L^{* 2}$. Now there exists the following exact sequence about the Galois cohomology (Inflation-Restriction sequence)

$$
0 \rightarrow H^{1}\left(L / K, \Gamma \stackrel{\mathrm{Gal}(\bar{K} / L)}{\mathrm{Inf}} \longrightarrow H^{1}(K, \Gamma) \stackrel{\operatorname{Res}}{\longrightarrow} H^{1}(L, \Gamma),\right.
$$

where $\Gamma:=\{ \pm 1\}$. Because the action of $\operatorname{Gal}(\bar{K} / L)$ on $\Gamma$ is trivial, we see

$$
\begin{aligned}
H^{1}\left(L / K, \Gamma^{\mathrm{Gal}(\bar{K} / L)}\right) & \cong \operatorname{Hom}(\operatorname{Gal}(L / K), \mathbb{Z} / 2 \mathbb{Z}) \\
& \cong \mathbb{Z} / 2 \mathbb{Z} .
\end{aligned}
$$

Since $H^{1}(K, \Gamma) \cong K^{*} / K^{* 2}$ and $H^{1}(L, \Gamma) \cong L^{*} / L^{* 2}$ by the Kummer theory, we obtain

$$
\mathbb{Z} / 2 \mathbb{Z} \rightarrow K^{*} / K^{* 2} \rightarrow L^{*} / L^{* 2},
$$

where the left map is given by that 1 maps to $-1 \bmod K^{* 2}$. Therefore the condition $-\alpha \in L^{* 2}$ implies that $\alpha$ or $-\alpha$ is contained in $K^{* 2}$. We assume the latter and lead contradiction. Let $\delta$ be an element of $K$ satisfying $-\alpha=\delta^{2}$. Because $-\beta$ is equal to $\delta^{2} / \lambda$ (recall $\lambda=\alpha / \beta \in K^{* 2}$ ), the elliptic curve $E$ is given by $y^{2}=x\left(x+\delta^{2}\right)\left(x+\delta^{2} / \lambda\right)$. Thus $E$ is isomorphic to $E^{\prime}: y^{2}=x(x+1)(x+\lambda)$ over $K$. This elliptic curve $E^{\prime}$ has bad (additive) reduction over $K$, because its $(L / K)$-twist $y^{2}=x(x-1)(x-\lambda)$ has good ordinary reduction over $K$ by Lemma 2.1. This contradicts our assumption that $E$ has good reduction.

Next we show that, for an ordinary curve $\tilde{E}$ defined over $\mathbb{F}$, there exists an element $\mu^{\uparrow}$ of $K$ such that the elliptic curve $E_{(\mu \uparrow)^{2}}$ is the canonical lift of $\tilde{E}$, and that there exists a canonical way to choose such an element.

Proposition 3.2. Assume that $\tilde{E} / \mathbb{F}$ is an ordinary elliptic curve, and that the 2 -torsion points of $\tilde{E}$ are rational over $\mathbb{F}$. Then there exists a unique element $\mu^{\uparrow} \in F$ satisfying $v\left(\mu^{\uparrow}-1\right)=3$ and $\left(\mu^{\uparrow}\right)^{\sigma}=\left(\mu^{\uparrow}+1\right) / 2 \sqrt{\mu^{\uparrow}}$ such that the canonical lift of $\tilde{E} / \mathbb{F}$ is given by the elliptic curve $E_{(\mu \uparrow)^{2}}$, where $\sigma$ is the Frobenius element in $\operatorname{Gal}\left(F / \mathbb{Q}_{2}\right)$.

Proof. The proof is given as follows:

(i) All 2-torsion points of the canonical lift of the ordinary curve $\tilde{E}$ are rational over $F$. Moreover there exists an element $\mu^{\uparrow} \in F$ satisfying $v\left(\mu^{\uparrow}-1\right)=3$ such that the elliptic curve $E_{(\mu \uparrow)^{2}}$ is the canonical lift of $\tilde{E}$. Such the elements are only $\mu^{\uparrow}$ and $1 / \mu^{\uparrow}$.

(ii) Let $\mu^{\uparrow} \in F$ be one of two choices given by (i). Then $\left(\mu^{\uparrow}\right)^{\sigma}$ equals to either $\left(\mu^{\uparrow}+1\right) /\left(2 \sqrt{\mu^{\uparrow}}\right)$ or $\left(2 \sqrt{\mu^{\uparrow}}\right) /\left(\mu^{\uparrow}+1\right)$.

First we give a proof of (i). For an ordinary elliptic curve $\tilde{E} / \mathbb{F}$, let $E / F$ be the canonical lift of $\tilde{E}$ and $\hat{E}$ the formal group of $E$. Because $E$ is the canonical lift of $\tilde{E}$, we have the split exact sequence

$$
0 \longrightarrow \hat{E}(\mathfrak{m})[2] \longrightarrow E(F)[2] \longrightarrow \tilde{E}(\mathbb{F})[2] \longrightarrow 0,
$$

where $\mathfrak{m}$ is the valuation ideal of $F$. Hence $E(F)[2] \cong \hat{E}(\mathfrak{m})[2] \oplus \tilde{E}(\mathbb{F})[2]$. Since we have assumed $\tilde{E}(\mathbb{F})[2] \cong \mathbb{Z} / 2 \mathbb{Z}$, it suffices to show that $\hat{E}(\mathfrak{m})[2] \cong \mathbb{Z} / 2 \mathbb{Z}$. We have the duplication formula for $\hat{E}(\mathfrak{m})([10$, Chapter 4])

$$
[2](X)=2 X+X^{2} h(X) \quad \text { for some } h(X) \in R \llbracket X \rrbracket,
$$


where $\mathrm{X}$ is a local parameter of $E$. Thus it suffices to prove that $f(X):=[2](X) / X=0$ has a solution in $\mathfrak{m}$. By the $p$-adic Weierstrass preparation theorem, the series $f(X)$ is expressed as

$$
f(X)=2^{m} P(X) U(X) \text { for some } m \in \mathbb{Z}_{\geq 0},
$$

where $P(X)$ is a monic polynomial in $R[X]$ with $P(X) \equiv X^{\operatorname{deg} P}(\bmod 2)$ and $U(X)$ belongs to the unit group of $R \llbracket X \rrbracket$. Note that $f(X)$ has just one root in an algebraic closure of $F$ because $E$ has good ordinary reduction, thus $P(X)$ is of degree one. Therefore $P(X)$ has a solution in $\mathfrak{m}$, and we see $\hat{E}(\mathfrak{m})[2] \cong \mathbb{Z} / 2 \mathbb{Z}$. This implies that $E$ is $F$-isomorphic to the curve defined by $y^{2}=x(x-\alpha)(x-\beta)$, where $\alpha, \beta$ are elements of $F$ and the point $(0,0)$ lies in the kernel of reduction map. By Proposition 3.1, it follows that $v(\alpha / \beta-1)$ equals 4 and the elements $\alpha$ and $\beta$ are square in $F$, thus $E$ is also $F$ isomorphic to $E_{\left(\mu^{\uparrow}\right)^{2}}$ and $E_{(1 / \mu \uparrow)^{2}}$, where $\left(\mu^{\uparrow}\right)^{2}=\alpha / \beta$ and $v\left(\mu^{\uparrow}-1\right)=3$. Moreover only $\mu^{\uparrow}$ and $1 / \mu^{\uparrow}$ satisfy the condition $v(v-1)=3$ with $E \cong E_{v^{2}}$ because $E$ is isomorphic to $E_{v^{2}}$ if and only if

$$
v^{2} \in\left\{\left(\mu^{\uparrow}\right)^{2}, \frac{1}{\left(\mu^{\uparrow}\right)^{2}}, 1-\left(\mu^{\uparrow}\right)^{2}, \frac{1}{1-\left(\mu^{\uparrow}\right)^{2}}, 1-\frac{1}{\left(\mu^{\uparrow}\right)^{2}}, \frac{\left(\mu^{\uparrow}\right)^{2}}{\left(\mu^{\uparrow}\right)^{2}-1}\right\} .
$$

Next we prove (ii). Put $\lambda:=\left(\mu^{\uparrow}\right)^{2}$. A consequence of Theorem 2.2 is that there exists a 2-isogeny $\phi: E_{\lambda} \rightarrow E_{\lambda^{\sigma}}$ such that the following diagram commutes:

$$
\begin{array}{cccc}
E_{\lambda}(\bar{K}) & \stackrel{\phi}{\longrightarrow} & E_{\lambda^{\sigma}}(\bar{K}) \\
\operatorname{red} \downarrow & & \text { red } \downarrow \\
\tilde{E}(\overline{\mathbb{F}}) & \stackrel{\mathrm{Fr}_{2}}{\longrightarrow} & \tilde{E}^{(2)}(\overline{\mathbb{F}}) .
\end{array}
$$

On the other hand, taking $\mu^{\prime \uparrow}:=\left(\mu^{\uparrow}+1\right) /\left(2 \sqrt{\mu^{\uparrow}}\right)$ and $\lambda^{\prime}:=\left(\mu^{\prime \uparrow}\right)^{2}$, we have also the following diagram because of Lemma 2.1:

$$
\begin{array}{ccc}
E_{\lambda}(\bar{K}) & \stackrel{g^{\uparrow}}{\longrightarrow} & E_{\lambda^{\prime}}(\bar{K}) \\
\text { red } \downarrow & & \text { red } \downarrow \\
\tilde{E}(\overline{\mathbb{F}}) & \stackrel{\mathrm{Fr}_{2}}{\longrightarrow} & \tilde{E}^{(2)}(\overline{\mathbb{F}})
\end{array}
$$

where $g^{\uparrow}$ is the isogeny given by replacing $\mu$ with $\mu^{\uparrow}$ in (2.1). Comparing (3.6) with (3.7), we see that

$$
E_{\lambda^{\sigma}} \cong E_{\lambda} / \Gamma \cong E_{\lambda^{\prime}},
$$

where $\Gamma:=\{O,(0,0)\} \subset E_{\lambda}(\bar{K})$. The isomorphism $E_{\lambda^{\sigma}} \rightarrow E_{\lambda^{\prime}}$ is given by $(x, y) \mapsto\left(u^{2} x, u^{3} y\right)$ for some $u \in F^{*}$ because $v\left(\lambda^{\sigma}-1\right)=v\left(\lambda^{\prime}-1\right)=4$. Therefore we see that $\left(\mu^{\uparrow}\right)^{\sigma}$ equals either $\left(\mu^{\uparrow}+1\right) /\left(2 \sqrt{\mu^{\uparrow}}\right)$ or $\left(2 \sqrt{\mu^{\uparrow}}\right) /\left(\mu^{\uparrow}+1\right)$.

\section{Proof of Theorem}

We start proving Theorem 1.1. Note that the elliptic curve $E_{n}:=E_{\mu_{n}^{2}}$ has good ordinary reduction over $K$ for each $n$, and that all 2-torsion points of the reduced curve $\tilde{E}_{0}$ are rational over $\mathbb{F}$. By applying Proposition 3.2 to $\tilde{E}_{0}$, there exists a unique element $\mu_{0}^{\uparrow} \in F$ satisfying

- $v\left(\mu_{0}^{\uparrow}-1\right)=3$,

- $\left(\mu_{0}^{\uparrow}\right)^{\sigma}=\left(\mu_{0}^{\uparrow}+1\right) /\left(2 \sqrt{\mu_{0}^{\uparrow}}\right)$,

- $E_{\left(\mu_{0}^{\uparrow}\right)^{2}}$ is the canonical lift of $\tilde{E}_{0}$,

where $\sigma$ is the Frobenius element in $\operatorname{Gal}\left(F / \mathbb{Q}_{2}\right)$. We define inductively

$$
\mu_{i+1}^{\uparrow}:=\frac{\mu_{i}^{\uparrow}+1}{2 \sqrt{\mu_{i}^{\uparrow}}} \quad(i \geq 0) .
$$

Then we obtain $\mu_{i+1}^{\uparrow}=\left(\mu_{i}^{\uparrow}\right)^{\sigma}$ because of the definition of $\mu_{0}^{\uparrow}$, thus it follows that

$$
\mu_{i+d}^{\uparrow}=\left(\mu_{i}^{\uparrow}\right)^{\sigma^{d}}=\mu_{i}^{\uparrow} \text {. }
$$

By $v\left(\mu_{n}-1\right)=3$ and $v\left(\mu_{i}^{\uparrow}-1\right)=3$, we may put

$$
\begin{array}{ll}
\mu_{n}=1+2^{3} \alpha_{n} & \text { for all } n \geq 0, \\
\mu_{i}^{\uparrow}=1+2^{3} \alpha_{i}^{\uparrow} & \text { for each } 0 \leq i \leq d-1,
\end{array}
$$

where $\alpha_{n}$ and $\alpha_{i}^{\uparrow}$ belong to the unit group of the integer ring of $K$. It is sufficient to show that the new sequence $\left\{\alpha_{d n+i}\right\}_{n \geq 0}$ is convergent to $\alpha_{i}^{\uparrow}$ for each $i$. Note that $\tilde{E}_{0} \cong \tilde{E}_{0}^{\uparrow}$ implies $\alpha_{0} \equiv \alpha_{0}^{\uparrow}(\bmod \pi)$, where $\pi$ is a prime element of $K$. It follows from the fact that the elliptic curves over $\mathbb{F}$

$$
y^{2}+x y=x^{3}+c, y^{2}+x y=x^{3}+c^{\prime}
$$

are isomorphic to each other if and only if $c=c^{\prime}$, and that $B$ and $B^{\prime}$ defined as in the proof of Lemma 2.1 for $\mu$ and $\mu^{\prime}$, 
respectively, are congruent modulo $\pi$ if and only if $\alpha \equiv \alpha^{\prime}(\bmod \pi)$, where $\mu=1+2^{3} \alpha$ and similarly for $\alpha^{\prime}$.

Now put $B(X, Y):=\left(1+2^{3} X\right) Y+2^{2}\left(1+2^{3} X\right) Y^{2}-X^{2} \in \mathbb{Z}[X, Y]$. (The reason for this definition will be explained later.) The following proposition implies Theorem 1.1, since we have $\alpha_{0} \equiv \alpha_{0}^{\uparrow}(\bmod \pi)$.

Proposition 4.1. Assume that there exists a positive integer $k \in \mathbb{N}$ such that $\alpha_{d n+i} \equiv \alpha_{i}^{\uparrow}\left(\bmod \pi^{k}\right)$ for some $n, i \geq 0$. Then there exists a unique element $\beta \in K$ such that $B\left(\alpha_{d n+i}, \beta\right)=0$ and $\beta \equiv \alpha_{i+1}^{\uparrow}\left(\bmod \pi^{k}\right)$. Moreover it follows that $\beta=\alpha_{d n+i+1}$ and $\beta \equiv \alpha_{i+1}^{\uparrow}\left(\bmod \pi^{k+1}\right)$.

Proof. This proof is given by applying [6, section 5] and [13, section 2] to our ramified case. The polynomial $B(X, Y) \in \mathbb{Z}[X, Y]$ has the following properties for each $n, i \geq 0$ :

$$
\begin{array}{ll}
B\left(\alpha_{n}, \alpha_{n+1}\right)=0, & \frac{\partial B}{\partial X}(X, Y) \equiv 0(\bmod \pi), \\
B\left(\alpha_{i}^{\uparrow}, \alpha_{i+1}^{\uparrow}\right)=0, & \frac{\partial B}{\partial Y}(X, Y) \equiv 1(\bmod \pi) .
\end{array}
$$

We may assume that there exists a positive integer $k \in \mathbb{N}$ such that $\alpha_{n} \equiv \alpha_{i}^{\uparrow}\left(\bmod \pi^{k}\right)$ for some $n, i \geq 0$. The existence of the unique element $\beta \in K$ satisfying $B\left(\alpha_{n}, \beta\right)=0$ and $\beta \equiv \alpha_{i+1}^{\uparrow}\left(\bmod \pi^{k}\right)$ follows immediately by applying Hensel's lemma to $B\left(\alpha_{n}, Y\right)$ due to the condition (4.1). We see that one of solutions of $B\left(\alpha_{n}, Y\right)=0$ is either $\alpha_{n+1}$ or $\left(-1-4 \alpha_{n+1}\right) / 4$. The latter is not in the integer ring of $K$, which implies $\beta$ should be $\alpha_{n+1}$.

In order to show $\alpha_{n+1} \equiv \alpha_{i+1}^{\uparrow}\left(\bmod \pi^{k+1}\right)$, we write the Taylor expansion of $B(X, Y)$ around $X=\alpha_{i}^{\uparrow}, Y=\alpha_{i+1}^{\uparrow}$ :

$$
B(X, Y)=B\left(\alpha_{i}^{\uparrow}, \alpha_{i+1}^{\uparrow}\right)+\left(X-\alpha_{i}^{\uparrow}\right) \frac{\partial B}{\partial X}(X, Y)+\left(Y-\alpha_{i+1} \uparrow\right) \frac{\partial B}{\partial Y}(X, Y)+\cdots .
$$

Substituting $X=\alpha_{n}$ and $Y=\alpha_{n+1}$, we obtain

$$
0 \equiv\left(\alpha_{n}-\alpha_{i}^{\uparrow}\right) \frac{\partial B}{\partial X}\left(\alpha_{n}, \alpha_{n+1}\right)+\left(\alpha_{n+1}-\alpha_{i+1}^{\uparrow}\right) \frac{\partial B}{\partial Y}\left(\alpha_{n}, \alpha_{n+1}\right)\left(\bmod \pi^{k+1}\right) .
$$

Since we have $\alpha_{n} \equiv \alpha_{i}^{\uparrow}\left(\bmod \pi^{k}\right)$ and the condition (4.1), the right hand side should be congruent to $\alpha_{n+1}-\alpha_{i+1}^{\uparrow}\left(\bmod \pi^{k+1}\right)$, thus we are done.

Remark. The polynomial $B(X, Y) \in \mathbb{Z}[X, Y]$ is constructed as follows: We set $A(X, Y):=4 X Y^{2}-(X+1)^{2}$. Then $A\left(\mu_{n}, \mu_{n+1}\right)=0$ for each $n \geq 0$, and $A\left(\mu_{i}^{\uparrow}, \mu_{i+1}^{\uparrow}\right)=0$ for each $i \geq 0$. Now we put $B^{\prime}(X, Y)=A\left(1+2^{3} X, 1+2^{3} Y\right)$. By removing common factors of $B^{\prime}(X, Y)$, we obtain $B(X, Y) \in \mathbb{Z}[X, Y]$. Therefore it is natural that the polynomial $B(X, Y)$ has the properties (4.1).

\section{Concluding Remarks}

In the rest of this paper, we give an explanation for a relation between the 2-adic arithmetic-geometric mean and elliptic curves defined over a 2-adic fields. Before we start to present it, we prove the following proposition which implies that the type of the reduction of an elliptic curve $E_{\lambda}$ is determined by the valuation of the element $\lambda$. We write $v$ for the unique extension of the valuation of $K$ to $\bar{K}$.

Proposition 5.1. Let $E / \bar{K}$ be an elliptic curve.

1. E has good ordinary reduction if and only if there exists an element $\lambda \in \bar{K}$ satisfying $v(\lambda-1)=4$ such that $E \cong E_{\lambda}$.

2. E has bad multiplicative reduction if and only if there exists an element $\lambda \in \bar{K}$ satisfying $v(\lambda-1)>4$ such that $E \cong E_{\lambda}$.

3. E has good supersingular reduction if and only if there exists an element $\lambda \in \bar{K}$ satisfying $0<v(\lambda-1)<4$ or $v(\lambda)=v(\lambda-1)=0$ such that $E \cong E_{\lambda}$.

Proof. Every elliptic curve defined over $\bar{K}$ is isomorphic to the Legendre form given by (1.3), and the type of the reduction is completely determined by the valuation of the $j$-invariant because the only supersingular elliptic curve in characteristic 2 is the curve with $j$-invariant 0 . Therefore we obtain

(i) $E_{\lambda}$ has good ordinary reduction $\Leftrightarrow v\left(j\left(E_{\lambda}\right)\right)=0$,

(ii) $E_{\lambda}$ has multiplicative reduction $\Leftrightarrow v\left(j\left(E_{\lambda}\right)\right)<0$,

(iii) $E_{\lambda}$ has supersingular reduction $\Leftrightarrow v\left(j\left(E_{\lambda}\right)\right)>0$, where

$$
v\left(j\left(E_{\lambda}\right)\right)=8+3 v\left((\lambda-1)^{2}+\lambda\right)-2 v(\lambda)-2 v(\lambda-1) .
$$

Therefore to check the type of the reduction of $E_{\lambda}$, it suffices to examine $v(\lambda)$ and $v(\lambda-1)$.

In the case $v(\lambda-1)=v(\lambda)=0$, the equation (5.1) becomes 


$$
v\left(j\left(E_{\lambda}\right)\right)=8+3 v\left((\lambda-1)^{2}+\lambda\right)>0,
$$

which means $E_{\lambda}$ has supersingular reduction by (iii).

All the other cases are reduced to the case $v(\lambda-1)>0$, because for any $\lambda \in \bar{K} \backslash\{0,1\}$ the elliptic curve $E_{\lambda}$ is isomorphic to the curve $E: y^{2}=x(x-1)(x-v)$ if and only if

$$
v \in\left\{\lambda, \frac{1}{\lambda}, 1-\lambda, \frac{1}{1-\lambda}, 1-\frac{1}{\lambda}, \frac{\lambda}{\lambda-1}\right\} .
$$

In the case $v(\lambda-1)>0$, the equation (5.1) becomes

$$
v\left(j\left(E_{\lambda}\right)\right)=8-2 v(\lambda-1) .
$$

By comparing with (i), (ii), and (iii), Proposition 5.1 is proved.

By Theorem 1.1, we can consider how the moduli of elliptic curves with good ordinary reduction over a 2-adic field behaves through the 2 -adic arithmetic-geometric mean. Indeed, when an elliptic curve $E$ having good ordinary reduction over $K$ is given, there exists, replacing $K$ with a finite extension, $\mu \in K$ satisfying $v(\mu-1)=3$ such that $E$ is isomorphic to $E_{\mu^{2}}$ over $K$ because of Proposition 5.1. Let $\left\{\mu_{n}\right\}_{n \geq 0}$ be the sequence of the 2-adic arithmetic-geometric mean for the element $\mu$, that is, the sequence $\left\{\mu_{n}\right\}_{n \geq 0}$ is defined by

$$
\mu_{0}:=\mu, \quad \mu_{n+1}=\frac{\mu_{n}+1}{2 \sqrt{\mu_{n}}},
$$

which consists of the ratio of the sequences of the 2-adic arithmetic-geometric mean of 1 and $\mu$. Then Theorem 1.1 implies that the $j$-invariant of $E_{\mu_{n}^{2}}$ are periodically attracted by that of the canonical lift, that is, the $j$-invariant of the canonical lift behaves as an attracting periodic point.

We can also consider the case of elliptic curves having multiplicative reduction by an argument similar to that for those with good ordinary reduction. When an elliptic curve $E$ having multiplicative reduction over $K$ is given, we may assume that there exists an element $\mu \in K$ satisfying $v(\mu-1)>3$ such that $E$ is isomorphic to $E_{\mu^{2}}$ over $K$. Let $\left\{\mu_{n}\right\}_{n \geq 0}$ be the sequence of the 2-adic arithmetic-geometric mean for the element $\mu$. The sequence $\left\{\mu_{n}\right\}_{n \geq 0}$ is convergent to 1 , that is, the family of elliptic curves $\left\{E_{\mu_{n}^{2}}\right\}_{n \geq 0}$ approaches to the singular curve $y^{2}=x(x-1)^{2}$. Therefore we see that the point at infinity is an attracting point on the $j$-plane.

Finally we deal with the case of elliptic curves having supersingular reduction. If an elliptic curve $E_{\mu^{2}}$ defined over $\bar{K}$ has supersingular reduction, we may assume that the element $\mu \in \bar{K}$ satisfies $0<v\left(\mu^{2}-1\right)<4$ or $v(\mu)=v(\mu-1)=0$. Let $\left\{\mu_{n}\right\}_{n \geq 0}$ be the sequence of the 2-adic arithmetic-geometric mean for the element $\mu$. However, in this case we have no canonical square root of $\mu$, that is, unlike the other cases we cannot define the canonical sequence $\left\{\mu_{n}\right\}_{n \geq 0}$ for $\mu$. It implies that there exist infinitely many choices for the sequence $\left\{\mu_{n}\right\}_{n \geq 0}$. The following lemma gives that for any such choice the sequence $\left\{\mu_{n}\right\}_{n \geq 0}$ is divergent, and that the valuation of the $j$ invariant of $E_{\mu_{n}^{2}}$ approaches the boundary of that of elliptic curves with supersingular reduction. Namely, the $j$-invariant of elliptic curves with supersingular reduction never be attracted by any points, although there exist no points like repelling fixed points. The lemma is proved by an easy estimate of the valuation of $\mu_{n}$, thus we omit the proof.

Lemma 5.2. Let $\mu$ be an element of $\bar{K}$ satisfying $0<v\left(\mu^{2}-1\right)<4$ or $v(\mu)=v(\mu-1)=0$, and let $\left\{\mu_{n}\right\}_{n \geq 0}$ be one of infinitely many sequences for the element $\mu$. Then there exists $N \in \mathbb{N}$ such that

$$
v\left(\mu_{n+1}\right)=\frac{v\left(\mu_{n}\right)}{2}-1<0 \text { for all } n \geq N .
$$

In particular, the union $\bigcup_{n \geq 0} K\left(\mu_{n}\right)$ is an infinite extension of $K$, and

$$
v\left(j\left(E_{\mu_{n}^{2}}\right)\right) \rightarrow 0(\text { as } n \rightarrow \infty) .
$$

The situation is summarized by Figure 1 .

\section{Appendix A: $\quad$ Periods of Elliptic Curves over $\mathbb{R}$}

In this appendix we introduce a way of calculating periods of an elliptic curve defined over the real number field by using the arithmetic-geometric mean.

Let $\left\{a_{n}\right\}_{\geq 0}$ and $\left\{b_{n}\right\}_{\geq 0}$ be the sequences of the arithmetic-geometric mean of $a$ and $b$ defined in (1.1), where $a$ and $b$ are positive real numbers satisfying $b>a$. We define an elliptic curve $E_{n}$ over $\mathbb{R}$ for each $n \geq 0$ by

$$
E_{n}: y^{2}=x(x-1)\left(x-\left(a_{n} / b_{n}\right)^{2}\right) \text {. }
$$

Then there exists the isogeny $g_{n}: E_{n} \rightarrow E_{n+1}$ of degree two defined in (2.1). Hence we obtain the diagram 


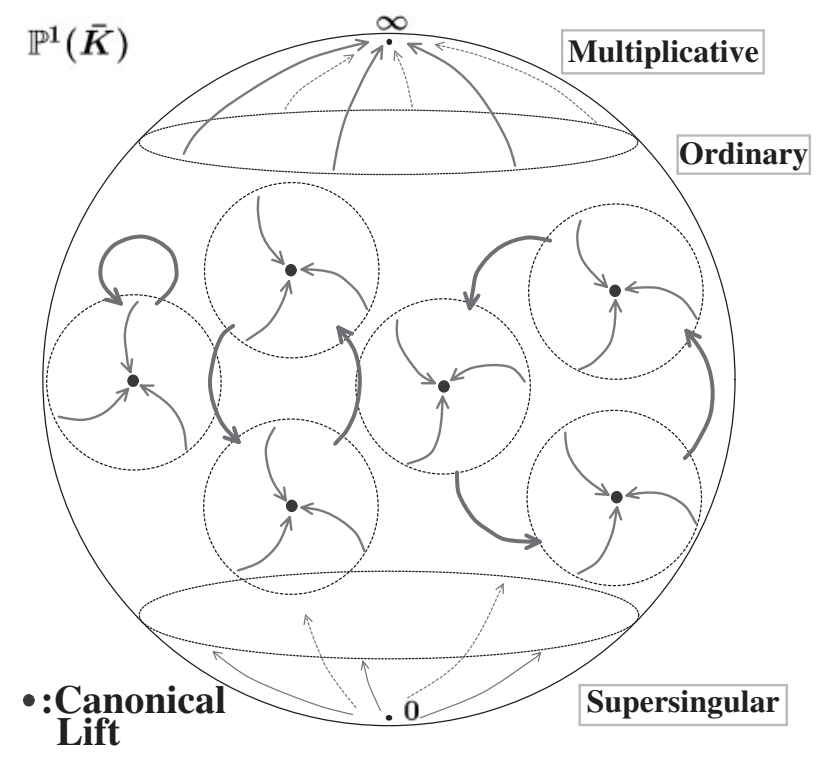

Fig. 1.

$$
E_{0} \stackrel{g_{0}}{\rightarrow} E_{1} \stackrel{g_{1}}{\rightarrow} \cdots \stackrel{g_{n-1}}{\rightarrow} E_{n} \stackrel{g_{n}}{\rightarrow} \cdots
$$

We employ (A.1) to calculate the periods of $E_{0}$. The idea is to relate them with that of a singular curve $E_{\infty}$ defined by

$$
E_{\infty}: y^{2}=x(x-1)^{2} .
$$

We regard $E_{\infty}$ as the limit of the family $\left\{E_{n}\right\}$ because the sequences $\left\{a_{n}\right\}$ and $\left\{b_{n}\right\}$ have the common limit. Since $E_{\infty}$ is a rational curve, its period can be calculated with elementary functions. Now, let $\alpha_{n}$ and $\beta_{n}$ be the real and imaginary basis of $H_{1}\left(E_{n}(\mathbb{C}), \mathbb{Z}\right)$, respectively (unique up to the sign). Note that $\alpha_{n}$ and $\beta_{n}$ satisfy

$$
g_{n *} \alpha_{n}=\alpha_{n+1}, \quad g_{n *} \beta_{n}=2 \beta_{n+1},
$$

where $g_{n_{*}}: H_{1}\left(E_{n}(\mathbb{C}), \mathbb{Z}\right) \rightarrow H_{1}\left(E_{n+1}(\mathbb{C}), \mathbb{Z}\right)$. Let $\omega_{n}=d x / y$ be a basis of $H^{0}\left(E_{n}, \Omega_{E_{n}}^{1}\right)$. The complex numbers $\int_{\alpha_{n}} \omega_{n}$ and $\int_{\beta_{n}} \omega_{n}$ are called the real and imaginary periods of the elliptic curve $E_{n}$, respectively.

Theorem A.1 (well-known). The periods of $E_{0}$ are given by

$$
\begin{aligned}
\int_{\beta_{0}} \omega_{0} & =\frac{2 \pi i}{M\left(a_{0} / b_{0}, 1\right)}, \\
\int_{\alpha_{0}} \omega_{0} & =\frac{2 \pi}{M\left(\sqrt{1-\left(a_{0} / b_{0}\right)^{2}}, 1\right)} .
\end{aligned}
$$

Proof. Since we have

$$
g_{n}^{*} \omega_{n+1}=2 \sqrt{\frac{a_{n}}{b_{n}}} \omega_{n},
$$

we see

$$
\int_{\beta_{n+1}} \omega_{n+1}=\frac{1}{2} \int_{g_{n *} \beta_{n}} \omega_{n+1}=\frac{1}{2} \int_{\beta_{n}} g_{n}^{*} \omega_{n+1}=\sqrt{\frac{a_{n}}{b_{n}}} \int_{\beta_{n}} \omega_{n} .
$$

Therefore we obtain

$$
\int_{\beta_{n+1}} \omega_{n+1}=\sqrt{\frac{a_{n}}{b_{n}}} \cdot \sqrt{\frac{a_{n-1}}{b_{n-1}}} \cdots \sqrt{\frac{a_{0}}{b_{0}}} \cdot \int_{\beta_{0}} \omega_{0}=\frac{b_{n+1}}{b_{0}} \int_{\beta_{0}} \omega_{0} .
$$

The right hand side of (A-2) converges to

$$
\frac{M\left(a_{0}, b_{0}\right)}{b_{0}} \int_{\beta_{0}} \omega_{0},
$$

where $M\left(a_{0}, b_{0}\right)$ is the common limit of the sequences $\left\{a_{n}\right\}$ and $\left\{b_{n}\right\}$. On the other hand, the left hand side of (A.2) is seen to converge to the "period of $E_{\infty}$ " 


$$
\int_{\beta_{\infty}} \frac{d x}{y}=2 \int_{0}^{-\infty} \frac{d x}{\sqrt{x(x-1)^{2}}}=2 \pi i
$$

Therefore we see

$$
\int_{\beta_{0}} \omega_{0}=\frac{2 \pi i}{M\left(a_{0} / b_{0}, 1\right)} .
$$

To calculate the other period, we consider an elliptic curve $E_{n}^{t}$ for each $n \geq 0$ defined by

$$
E_{n}^{t}: y^{2}=x(x-1)\left(x-\left(1-\left(a_{n} / b_{n}\right)^{2}\right)\right) .
$$

This curve is a twist of $E_{n}$ because we have an isomorphism

$$
\phi_{n}: E_{n}(\mathbb{C}) \ni(x, y) \mapsto(1-x, i y) \in E_{n}^{t}(\mathbb{C}) .
$$

Note that there exists an imaginary basis $\beta_{n}^{t}$ of $H_{1}\left(E_{n}^{t}, \mathbb{Z}\right)$ such that $\phi_{n *} \alpha_{n}=\beta_{n}^{t}$. By a way similar to the imaginary period of $E_{0}$, we obtain

$$
\int_{\beta_{0}^{t}} \omega_{0}^{t}=\frac{2 \pi i}{M\left(\sqrt{1-\left(a_{0} / b_{0}\right)^{2}}, 1\right)},
$$

where $\omega_{0}^{t}=d x / y$ is a basis of $H^{0}\left(E_{0}^{t}, \Omega_{E_{0}^{t}}^{1}\right)$. Now we see that

$$
\int_{\alpha_{0}} \omega_{0}=\frac{1}{i} \int_{\phi_{0_{*} \alpha}} \omega_{0}^{t}=-i \int_{\beta_{0}^{t}} \omega_{0}^{t} .
$$

Therefore we obtain

$$
\int_{\alpha_{0}} \omega_{0}=\frac{2 \pi}{M\left(\sqrt{1-\left(a_{0} / b_{0}\right)^{2}}, 1\right)} .
$$

\section{Appendix B: Periods of the Tate Curve}

In this appendix we observe a relation between the Hodge-Tate periods of a Tate curve and the $p$-adic arithmeticgeometric mean by an argument similar to Appendix A. Throughout this section, let $K$ denote a finite extension of $\mathbb{Q}_{p}$ for a prime $p$ and $v$ the valuation of $K$ satisfying $v(p)=1$. We fix an algebraic closure $\bar{K}$ of $K$.

Let $a$ and $b$ be elements in $K$ satisfying $v((a / b)-1)>0$ if $p \neq 2$ (resp. $v((a / b)-1)>3$ if $p=2$ ). We also define the sequences $\left\{a_{n}\right\}_{n \geq 0}$ and $\left\{b_{n}\right\}_{n \geq 0}$, which is called the sequences of the $p$-adic arithmetic-geometric mean of $a$ and $b$,

$$
\begin{array}{ll}
a_{0}:=a, & b_{0}:=b, \\
a_{n+1}:=\frac{a_{n}+b_{n}}{2}, & b_{n+1}:=b_{n} \sqrt{\frac{a_{n}}{b_{n}}}(n \geq 0),
\end{array}
$$

where $\sqrt{a_{n} / b_{n}}$ is the square root of $a_{n} / b_{n}$ satisfying $v\left(\sqrt{a_{n} / b_{n}}-1\right)>0$ if $p \neq 2\left(\operatorname{resp} . v\left(\sqrt{a_{n} / b_{n}}-1\right)>2\right.$ if $\left.p=2\right)$. Then these sequences are convergent to a common limit $M(a, b)$. Henniart and Mestre proved the following result by using $p$-adic theta functions ([7]). We will give a new proof without using the theta functions.

Theorem B.1 (Henniart-Mestre). Let $a$ and $b$ be elements in $K$ satisfying $v((a / b)-1)>0$ if $p \neq 2$ (resp. $v((a / b)-1)>3$ if $p=2)$. Let $E_{0}$ be an elliptic curve defined by

$$
E_{0}: y^{2}=x(x-1)\left(x-(a / b)^{2}\right)
$$

(this curve has split multiplicative reduction over $K$ ), and let

$$
\phi_{0}: \bar{K}^{*} /\langle q\rangle \stackrel{\cong}{\rightarrow} E_{0}(\bar{K}), \quad q \in K
$$

be an isomorphism given by Theorem B.2 below. Define $u \in K^{*}$ by $\phi_{0}^{*}(d x / y)=u \cdot d t / t$, where $d x / y$ and $d t / t$ are the invariant differential forms of $E_{0}$ and $\bar{K}^{*} /\langle q\rangle$, respectively. Then

$$
u^{2}=M(a / b, 1)^{-2} .
$$

Theorem B.2 (Tate). Let $E$ be an elliptic curve with split multiplicative reduction over $K$ (called the Tate curve). Then there exists a unique element $q \in K$ satisfying $v(q)>0$ such that $\bar{K}^{*} /\langle q\rangle$ is isomorphic to $E(\bar{K})$. The isomorphism $\phi: \bar{K}^{*} /\langle q\rangle \stackrel{\cong}{\rightarrow} E(\bar{K})$ is unique up to the sign and commutes with $\mathrm{Gal}(\bar{K} / K)$-action. 
For the proof of Theorem B.2, see [11] or [12] for example.

Proof of Theorem B.1. We put $E_{n}: y^{2}=x(x-1)\left(x-\left(a_{n} / b_{n}\right)^{2}\right)$ for $n \geq 0$, where $\left\{a_{n}\right\}$ and $\left\{b_{n}\right\}$ are the sequences of the $p$-adic arithmetic-geometric mean of $a$ and $b$, and take a regular differential form $\omega_{n}:=d x / y$. Let $h_{n}: E_{n+1} \rightarrow E_{n}$ be the dual isogeny of $g_{n}$ defined in (2.1). Then we see $h_{n}^{*} \omega_{n}=\sqrt{b_{n} / a_{n}} \omega_{n+1}$. By induction, there exists an isomorphism $\phi_{n+1}: \bar{K}^{*} /\left\langle q^{2^{n+1}}\right\rangle \rightarrow E_{n+1}(\bar{K})$ satisfying

$$
\phi_{n+1}^{*} \omega_{n+1}=u \sqrt{\frac{a_{0}}{b_{0}}} \cdot \sqrt{\frac{a_{1}}{b_{1}}} \cdots \cdot \sqrt{\frac{a_{n}}{b_{n}}} \cdot d t / t=u \frac{b_{n+1}}{b_{0}} \cdot d t / t
$$

such that the following diagram is commutative:

$$
\begin{array}{ccc}
\bar{K}^{*} /\left\langle q^{2^{n+1}}\right\rangle & \stackrel{\text { Proj. }}{\longrightarrow} & \bar{K}^{*} /\left\langle q^{2^{n}}\right\rangle \\
\phi_{n+1} \downarrow & & \phi_{n} \downarrow \\
E_{n+1}(\bar{K}) & \stackrel{h_{n}}{\longrightarrow} & E_{n}(\bar{K}) .
\end{array}
$$

Let $E_{\infty}$ be a singular curve defined by

$$
E_{\infty}: y^{2}=x(x-1)^{2} \text {. }
$$

We regard this curve as the limit of the family $\left\{E_{n}\right\}_{n \geq 0}$ because the sequences $\left\{a_{n}\right\}_{n \geq 0}$ and $\left\{b_{n}\right\}_{n \geq 0}$ have the common limit. Let $\phi_{\infty}: \bar{K}^{*} \rightarrow E_{\infty} \backslash\{(1,0)\}$ be the isomorphism defined by

$$
\phi_{\infty}: t \mapsto\left(\frac{(1+t)^{2}}{(1-t)^{2}}, \frac{4 t(1+t)}{(1-t)^{3}}\right),
$$

which satisfies

$$
\phi_{\infty}^{*} \omega_{\infty}=\phi_{\infty}^{*}(d x / y)=d t / t
$$

On the other hand, the equation (B.2) implies

$$
\phi_{\infty}^{*} \omega_{\infty}= \pm u M(a / b, 1) \cdot d t / t .
$$

By comparing $(\mathrm{B} \cdot 3)$ and $(\mathrm{B} \cdot 4)$, we obtain the equation $(\mathrm{B} \cdot 1)$.

Next we introduce the Hodge-Tate periods of an elliptic curve $E$ defined over $K$, which is a $p$-adic analogue of the periods of an elliptic curve defined over $\mathbb{C}$. There exists the following bilinear pairing defined by Fontaine in [5]

$$
\langle,\rangle: T_{p}(E) \times H^{0}\left(E, \Omega_{E}^{1}\right) \rightarrow \mathbb{C}_{p}(1),
$$

where $T_{p}(E)$ is the Tate module of $E, H^{0}\left(E, \Omega_{E}^{1}\right)$ is the space of regular differential forms of $E, \mathbb{C}_{p}$ is the $p$-adic completion of the algebraic closure of $\mathbb{Q}_{p}$, and $\mathbb{C}_{p}(1)$ is the 1-st Tate twist of $\mathbb{C}_{p}$. The image of (B.5) is called the Hodge-Tate periods of $E$. One of the important properties of this pairing is the compatibility with isogenies of elliptic curves. Namely if $g: E \rightarrow E^{\prime}$ is an isogeny between elliptic curves, then it follows that

$$
\left\langle e_{E}, g^{*} \omega_{E^{\prime}}\right\rangle=\left\langle g_{*} e_{E}, \omega_{E^{\prime}}\right\rangle, \quad e_{E} \in T_{p}(E), \omega_{E^{\prime}} \in H^{0}\left(E^{\prime}, \Omega_{E^{\prime}}^{1}\right),
$$

where $g_{*}: T_{p}(E) \rightarrow T_{p}\left(E^{\prime}\right)$ is the map induced by the isogeny $g$. For details of the pairing (B.5), see [2], [3] or [5].

When $E$ is a Tate curve defined over $K$, we take an element $e_{E}$ of $T_{p}(E)$ as follows: Let $\phi: \bar{K}^{*} /\langle q\rangle \rightarrow E(\bar{K})$ be an isomorphism defined by Theorem B.2. Let $\mu_{m}$ denote the subset of $\bar{K}$ defined by $\mu_{m}=\left\{x \in \bar{K}: x^{m}=1\right\}$. Then we choose once and for all a compatible sequence of primitive $p^{n}$-th roots of unity, $\varepsilon^{(0)}=1$, and $\varepsilon^{(n)} \in \mu_{p^{n}}$, such that $\varepsilon^{(1)} \neq 1$ and $\left(\varepsilon^{(n+1)}\right)^{p}=\varepsilon^{(n)}$. Define $e_{E}=\phi_{*} e \in T_{p}(E)$, where $e:=\lim \varepsilon^{(n)} \in T_{p}\left(\bar{K}^{*} /\langle q\rangle\right)$. We remark that $e_{E}$ depends on the choice of $e$ and $\phi$.

Theorem B.3. Let $E_{0}$ and $\phi_{0}$ be the same as in Theorem B.1. Let $e_{E_{0}}$ be the element of $T_{p}\left(E_{0}\right)$ defined above. Put $i:=\varepsilon^{(2)}, \varpi:=\langle e, d t / t\rangle / 2 i \in \mathbb{C}_{p}(1)$, and $\omega_{E_{0}}:=d x / y \in H^{0}\left(E_{0}, \Omega_{E_{0}}^{1}\right)$. Then (up to the sign)

$$
\left\langle e_{E_{0}}, \omega_{E_{0}}\right\rangle=\frac{2 \varpi i}{M(a / b, 1)} \text {. }
$$

Proof.

$$
\begin{aligned}
\left\langle e_{E_{0}}, \omega_{E_{0}}\right\rangle & =\left\langle\phi_{0 *} e, \omega_{E_{0}}\right\rangle & & \text { the definition of } e_{E_{0}} \\
& =\left\langle e, \phi_{0}^{*}(d x / y)\right\rangle & & \text { by }(\mathrm{B} \cdot 6) \\
& =\left\langle e, \pm M(a / b, 1)^{-1} \cdot d t / t\right\rangle & & \text { from Theorem B.1 } \\
& = \pm M(a / b)^{-1} \cdot\langle e, d t / t\rangle . & &
\end{aligned}
$$


Remark. Theorem B.3 gives a part of $p$-adic analogue of Theorem A.1. Unfortunately we do not know how to compute $\left\langle f_{E_{0}}, \omega_{E_{0}}\right\rangle$ for $f_{E_{0}} \in T_{p}\left(E_{0}\right)$ which is linearly independent of $e_{E_{0}}$. Therefore in order to compute it by using the $p$-adic arithmetic-geometric mean, we will need to consider another argument.

\section{Acknowledgments}

The authors would like to thank Professor Takao Yamazaki for his continuous support and invaluable suggestions.

\section{REFERENCES}

[1] Carls, Robert, Generalized arithmetic geometric mean, Doctor's thesis, Rijks Universiteit Groningen (2004).

[2] Coleman, Robert, F., "Hodge-Tate periods and p-adic abelian integrals," Invent. Math., 78: 351-379 (1984).

[3] Colmez, Pierre, "Périodes p-adiques des variétés abéliennes," Math. Ann., 292: 629-644 (1992).

[4] Cox, David, A., “The arithmetic-geometric mean of Gauss," Enseign. Math. (2), 30: 275-330 (1984).

[5] Fontaine, Jean-Marc, "Formes différentielles et modules de Tate des variétés abéliennes sur les corps locaux," Invent. Math., 65: 379-409 (1981/82).

[6] Gaudry, Pierrick, A comparison and a combination of SST and AGM algorithms for counting points of elliptic curves in characteristic 2, Advances in cryptology-ASIACRYPT 2002, Lecture Notes in Comput. Sci., 2501, 311-327, Springer, Berlin (2002).

[7] Henniart, Guy, and Mestre, Jean-François, "Moyenne arithmético-géométrique p-adique," C. R. Acad. Sci. Paris Sér. I Math., 308: 391-395 (1989).

[8] Messing, William, The crystals associated to Barsotti-Tate groups: with applications to abelian schemes, Lecture Notes in Mathematics, Vol. 264, Springer-Verlag, Berlin (1972).

[9] Satoh, Takakazu, On $p$-adic point counting algorithms for elliptic curves over finite fields, Algorithmic number theory (Sydney, 2002), 43-66, Lecture Notes in Comput. Sci., 2369, Springer, Berlin (2002).

[10] Silverman, Joseph, H., The arithmetic of elliptic curves, Graduate Texts in Mathematics, 106, Springer-Verlag, New York (1986).

[11] Silverman, Joseph, H., Advanced topics in the arithmetic of elliptic curves, Graduate Texts in Mathematics, 151, SpringerVerlag, New York (1994).

[12] Tate, John, A review of non-Archimedean elliptic functions, Elliptic curves, modular forms, \& Fermat's last theorem (Hong Kong, 1993), Ser. Number Theory, I, 162-184, Int. Press, Cambridge, MA (1995).

[13] Vercauteren, Frederik, and Preneel, Bart, and Vandewalle, Joos, A memory efficient version of Satoh's algorithm, Advances in cryptology_EUROCRYPT 2001 (Innsbruck), Lecture Notes in Comput. Sci., 2045, 1-13, Springer, Berlin (2001). 